\title{
Anemia hemolítica como manifestación inicial de macroglobulinemia de Waldenström
}

\author{
Daniela Hernández-Islas ${ }^{1 *}$, Laura Elena Zamora-Cervantes ${ }^{1}$, Víctor Hugo García-López ${ }^{2}$, \\ Genaro Hernández-Pilotzi ${ }^{1}$, José Roberto Piña-Gorraez ${ }^{1}$, Nayel Xi Xendali Arista-Olvera ${ }^{1}$ \\ Y CÉsAR SÁNCHEZ-GonZÁLEZ ${ }^{1}$ \\ ${ }^{1}$ Residente de cuarto año de Medicina Interna; ${ }^{2}$ Jefe del Servicio de Medicina Interna. Hospital General Tláhuac, Ciudad de México, México
}

\begin{abstract}
RESUMEN
La macroglobulinemia de Waldenström (MW) es una neoplasia hematológica con proliferación linfoplasmocitaria, producción monoclonal de inmunoglobulina M (IgM) e infiltración medular. En México tiene una incidencia del $0.18 \%$. En este artículo se presenta el caso de un varón de 63 años con disnea, astenia, pérdida ponderal, ictericia, pancitopenia, electroforesis de proteínas (SPEP) con Rouleaux y Coombs directo con autoanticuerpos fríos. Se inicia protocolo diagnóstico de anemia hemolítica autoinmune, aspirado de médula ósea (MO) con células de Mott y algunas plasmáticas y biopsia de hueso con células plasmáticas anormales en un 15\%. SPEP con hipergammaglobulinemia y pico monoclonal $\gamma$, inmunofijación con IgM- $\lambda$ e inmunofijación en orina para proteína de Bence Jones negativa. Se concluye con diagnóstico de MW.
\end{abstract}

Palabras clave: Anemia hemolítica. Macroglobulinemia de Waldeström. Neoplasia hematológica.

\begin{abstract}
Waldenström macroglobulinemia (WM) is a hematological malignancy with lymphoplasmacytic proliferation, monoclonal production of immunoglobulin $\mathrm{M}(\mathrm{IgM})$ and spinal infiltration. In Mexico, it has an incidence of $0.18 \%$. This article presents the case of a 63-year-old boy with dyspnea, asthenia, weight loss, jaundice, pancytopenia, serum protein electrophoresis (SPEP) with Rouleaux and direct Coombs with cold autoantibodies. The protocol of autoimmune hemolytic anemia, bone marrow aspirate with Mott cells and some plasmatic and bone biopsy with abnormal plasma cells in 15\%. Serum protein electrophoresis with hypergammaglobulinemia and monoclonal peak, immunofixation with IgM-I and immunofixation in urine for Bence Jones protein negative. It concludes with the diagnosis of WM. (Hosp Med Clin Manag. 2018;11:148-54)

Corresponding author: Daniela Hernández-Islas, daniela.islas@outlook.com
\end{abstract}

Key words: Hemolytic anemia. Waldenström's macroglobulinemia. Hematological malignancy.

\section{Correspondencia:}

*Daniela Hernández-Islas

Servicio de Medicina Interna

Hospital General Ticomán

Plan de San Luis, $s / n$

Col. La Purísima Ticomán, Gustavo A. Madero

C.P. 07330, Ciudad de México, México

Date of reception: $19-06-2018$

E-mail: daniela.islas@outlook.com

Date of acceptance: 13-09-2018

Doi: 10.24875/HMCM.18000133 


\section{INTRODUCCIÓN}

La MW es una entidad clínico-patológica definida como un trastorno linfoproliferativo B poco común caracterizado por la infiltración de la médula ósea por células linfoides con patrón predominantemente intertrabecular, junto a la demostración de la presencia de una gammapatía monoclonal IgM. A día de hoy se desconoce su causa exacta, aunque se cree que es determinada por factores genéticos. Es una enfermedad poco frecuente: se diagnostica a tres millones de personas al año y representa un 1-2\% de los cánceres hematológicos. En México tiene una prevalencia del $0.18 \%$. Las manifestaciones clínicas pueden ser muy variadas e inespecíficas, y afectar a diversos órganos y sistemas, pudiendo también producir fenómenos autoinmunes, como anemia hemolítica. A pesar de que es una enfermedad incurable, el tratamiento es a base de quimioterapia con un solo fármaco o con combinaciones de varios. La supervivencia media de las personas que la padecen es de 5 a 10 años, teniendo en cuenta que la mortalidad se asocia con el propio cáncer, y no con otras complicaciones derivadas. Presentamos este caso porque se trata de una enfermedad poco frecuente e infradiagnosticada en nuestro país, y porque inicialmente se presentó con anemia hemolítica, fenómeno de presentación inicial también poco común en esta enfermedad.

\section{CASO CLÍNICO}

Varón de 63 años, originario de Oaxaca, residente de la Ciudad de México, casado, con escolaridad primaria, actualmente desempleado, aunque antes del padecimiento trabajaba como ayudante de albañil, y católico.

Antecedentes heredo familiares: madre finada por insuficiencia hepática alcohólica, carga genética paterna para cáncer de próstata.

Antecedentes personales no patológicos: habita en una vivienda con materiales perdurables; hacinamiento, positivo; zoonosis, negativo; baño diario; dieta hipercalórica; ingesta de lácteos no pasteurizados, negativo; vacunas recientes, negativo; viajes recientes, positivo, a Oaxaca; convivencia con tosedores crónicos, negativo.

Antecedentes personales patológicos: hemotipo O positivo; tabaquismo, positivo -IT 96 paquetes/año-, aunque actualmente suspendido; alcoholismo, positivo, con un patrón de unos 5 I a la semana durante 34 años a base destilados y fermentados, aunque actualmente suspendido; antecedentes cronicodegenerativos, infectocontagiosos, traumatológicos, transfusionales, alérgicos, quirúrgicos, hospitalizaciones, toxicomanías y exposición a biomasa, negativos.

Antecedentes androgénicos: Inicio de vida sexual activa, 15; Parejas sexuales, 8; heterosexuales; preservativo, 0\%; Infecciones de transmisión sexual, negativo; Antígeno prostático específico, negativo desde hace ocho meses.

El padecimiento empieza tres meses antes con disnea, inicialmente de grandes esfuerzos y que luego se vuelve progresiva, astenia, fatiga y adinamia. En un mes progresa a ortopnea y se agrega palidez en tegumentos, además de pérdida ponderal de $10 \mathrm{~kg}$ no intencional, motivo por cual acude a un médico particular, con el que inicia manejo con hematínicos, sin especificarse dosis. Tres semanas antes del ingreso presenta ictericia conjuntival, así como exacerbación de la sintomatología, hasta llegar a ser en reposo, por lo que acude a un centro de salud, donde se le realizan paraclínicos. Al encontrar una hemoglobina de $4.2 \mathrm{~g} / \mathrm{dl}$, se envía a nuestro nosocomio. El paciente ingresa en el Servicio de Urgencias con: TA, 90/40 mmHg; FC, 84 lpm; FR, 17 rpm, y glicemia capilar de $118 \mathrm{mg} / \mathrm{dl}$. Se realiza frotis sanguíneo, en el que se observa aglutinación. Ante la sospecha de anemia hemolítica autoinmune, es ingresado en el Servicio de Medicina Interna. En la exploración física se observa: IMC de $24.8 \mathrm{~kg} / \mathrm{m}^{2}$, neurológicamente íntegro, ictérico, sin lesiones en piel, adoncia parcial, sin adenomegalias, ruidos cardíacos rítmicos y sin soplos, tórax con murmullo vesicular generalizado sin agregados, abdomen plano, no doloroso, sin organomegalias, peristaltismo normoactivo, y genitales, extremidades y columna sin alteraciones.

Los datos de laboratorio iniciales muestran pancitopenia (leucocitos totales, $3.2 \times 10^{3} / \mathrm{ml}$; neutrófilos, $2 \times 10^{3} / \mathrm{ml}$; linfocitos, $0.810^{3} / \mathrm{ml}$; hemoglobina, $4.1 \mathrm{~g} / \mathrm{dl}$, y plaquetas, $7410^{3} / \mathrm{ml}$ ), anemia inicialmente macrocítica con VCM de $110 \mathrm{fl}, \mathrm{HbCM}$ de $39.7 \mathrm{pg}$, y reticulocitos corregidos al 5.3\%; hiperbilirrubinemia de $3.8 \mathrm{mg} / \mathrm{dl}$ con predominio indirecto, lactato deshidrogenasa de $136 \mathrm{UI} / \mathrm{I}$, AST de $35 \mathrm{UI} / \mathrm{I}$, ALT de $21 \mathrm{UI} / \mathrm{l}$, fosfatasa alcalina de $91 \mathrm{UI} / \mathrm{I}, \mathrm{GGT}$ de $21 \mathrm{UI} / \mathrm{l}$, proteínas totales de $8.1 \mathrm{~g} / \mathrm{dl}$ (albúmina, $3.7 \mathrm{~g} / \mathrm{dl}$; globulinas, $4.4 \mathrm{~g} / \mathrm{dl}$ ); TP de $15.1 \mathrm{~s}$; TTPa de $24.9 \mathrm{~s}$; INR de $1.36 \mathrm{~s}$; fibrinógeno de $270 \mathrm{mg} / \mathrm{dl}$; función 
renal conservada con creatinina de $0.8 \mathrm{mg} / \mathrm{dl}$, colesterol de $25 \mathrm{mg} / \mathrm{dl}$ y triglicéridos de $22 \mathrm{mg} / \mathrm{dl}$; sin alteraciones hidroelectrolíticas (calcio corregido por albúmina de $9 \mathrm{mg} / \mathrm{dl}$ ); hiperlactatemia de 3; examen general de orina con densidad de 1,016, proteinuria de $20 \mathrm{mg} / \mathrm{dl}$, sin glucosuria, bacteriuria, leucocituria o hematuria. Se realiza nuevo frotis de sangre periférica, en el que se observa células de Rouleaux.

Coombs directo positivo con título de 16, anticuerpos $\mathrm{C} 3 \mathrm{~d}$, con especificidad autoanti-I a $22^{\circ}$ (no se realiza fenotipo ante transfusión reciente en seis ocasiones). El aspirado de médula ósea es normocelular, los megacariocitos están moderadamente disminuidos, hay un ligero incremento de granulocitos adultos y ligera depresión granulocitaria juvenil, conservación eritroide y linfocitaria, con células de Mott y algunas plasmáticas. Biopsia de hueso infiltrada por células plasmáticas anormales en el $15 \%$ y linfocitos, solicitándose inmunohistoquímica para CD20, CD38, CD138 y CD10.

Panel viral con citomegalovirus, Epstein-Barr, varicela zóster, herpes 6, 7 y 8 , enterovirus, toxoplasma, parvovirus $\mathrm{B} 19$, rubeola, hepatitis $\mathrm{B}$ y $\mathrm{C}, \mathrm{VIH}$ no reactivos; herpes simple 1 IgM e IgG positivo, tipo 2 IgG positivo y PCR para ambos positivo. Niveles séricos de ácido fólico de $11 \mathrm{ng} / \mathrm{ml}(2.7-17 \mathrm{ng} / \mathrm{ml})$, vitamina B12 > 6,000 pg/ml (200-900), haptoglobina de $94.9 \mathrm{mg} / \mathrm{dl}$ (41-165), C3 y C4 bajos (35 y $<2.9 \mathrm{mg} / \mathrm{dl}$, respectivamente), anti-Ro, anti-La, anti-SM, anti-ADN, anticardiolipinas, anticoagulante lúpico, p-ANCA y c-ANCA, negativos.

En la electroforesis de proteínas en suero se observa: albúmina, $46.6 \%$; $\gamma, 38.4 \%$; $\beta, 4.9 \%$, y $\alpha 1$ del $2.3 \%$ y $\alpha 2$ del $7.8 \%$, con hipergammaglobulinemia con pico asimétrico en la región puente $\beta$-globulina y en la inmunofijación con componente IgM y restricción para $\lambda$. En la inmunofijación en orina la proteína de Bence Jones es negativa y la $\beta 2$-microglobulina ( $\beta 2-\mathrm{M}$ ) es de $2.87 \mathrm{mg} / \mathrm{l}$.

En la radiografía de cráneo no se observan lesiones líticas. Ultrasonido abdominal normal, tomografía simple y contrastada abdominal con engrosamiento gástrico, pero en la panendoscopia, pliegues gástricos con edema, eritema y erosiones, antro con eritema alternado con zonas blanquecinas con patrón geográfico, fondus con zonas de metaplasia intestinal; duodeno con patrón aterciopelado con placas blanquecinas sugerentes de atrofia vellosa; ileon terminal, colon y recto dentro de la normalidad. El reporte de la biopsia confirma metaplasia intestinal.

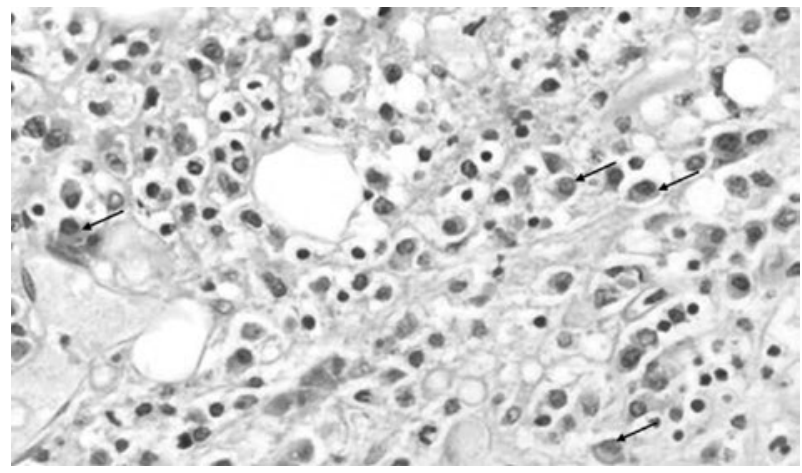

Figura 1. Biopsia de hueso tomada de patología. Tinción de H\&E. Aumento de 100 '. Se observa infiltrado de células plasmáticas anormales (flechas) y linfocitos.

Dada lo sospecha inicial de anemia hemolítica autoinmune, se inicia manejo con metilprednisolona (1 g por 3 días), seguido de prednisona en dosis de reducción. No hay mejoría de la anemia, presentando incluso 15 días después hemólisis, con descenso de $2 \mathrm{~g}$ de hemoglobina, bilirrubina total de 4.7 con indirecta de 3.6, lactato deshidrogenasa de 1,771 UI/l, así como lesión renal aguda con creatinina de hasta 5.2, urea de 158, anuria e hipercalemia. En consecuencia, se ameritó nueva terapia con esteroides, en esta ocasión dexametasona, y hemodiálisis. Posteriormente se confirmó bacteriemia e infección de las vías urinarias por Staphylococcus haemolyticus, por lo que se agrega a la terapia glucopéptido y fluoroquinolona. El paciente presentó mejoría en la cifra de hemoglobina, pero se perpetuó la lesión renal, por lo que se continuó con la terapia de sustitución renal. Cuarenta días después de la hospitalización se produce la defunción del paciente a causa de un choque séptico secundario a neumonía intrahospitalaria sin germen aislado. Dos días antes se obtuvo el resultado de electroforesis de proteínas con inmunofijación, así como el de patología de biopsia de hueso (Figs. 1-3).

\section{DISCUSIÓN}

El diagnóstico de la MW requiere: a) la presencia de gammapatía monoclonal IgM; b) infiltración de médula ósea por plasmocitosis medular y linfocitos pequeños; c) infiltración en hueso difuso, intersticial o nodular, y d) inmunofenotipo con Ig+, CD5-, CD10-, CD19+, CD20+ y CD23-. Es una enfermedad poco común y con manifestaciones clínicas variables ${ }^{1}$. La anemia se presenta en el $60-70 \%$ de los casos y generalmente es normocítica; 


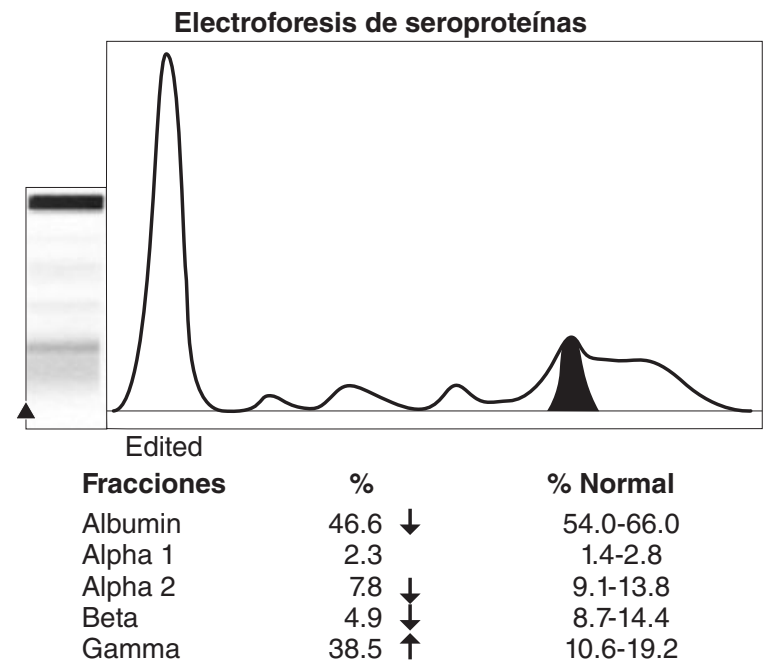

Figura 2. SPEP pico del 38.5\% en región $\gamma$.

en el menor de los casos se presenta como anemia hemolítica por producción de IgM con capacidad para ejercer autoinmunidad, al unirse a antígenos I o i eritrocitarios, activándose a temperaturas $<37^{\circ} \mathrm{C}$, entidad poco común en esta enfermedad².

De acuerdo con el primer panel de consenso del Workshop de Atenas en 2010, se excluyó la fijación de un umbral mínimo de lgM monoclonal en el suero para definir la enfermedad, hecho que facilitó el diagnóstico, ya que es uno de los puntos que más discordancias ha generado. El 90\% de los casos presentan gammapatía monoclonal IgM y menos del 5\%, IgA e IgM. Es una enfermedad poco frecuente. En EE.UU. se estima una incidencia anual de 2-5 casos por millón de habitantes; en México se desconocen la cifras exactas, aunque se tiene una incidencia del $0.18 \%$ de acuerdo al estudio realizado por Ruiz en $2000^{3}$. La MW afecta sobre todo a varones (70\%) de edad media y avanzada (> 55 años), y es considerada una etiología genética, pues se ha encontrado que alrededor del $83 \%$ de los pacientes con MW tienen anomalías cromosómicas; en más del $90 \%$ de los pacientes se ha encontrado mutación somática sin mutación somática L265P del gen MYD88 en el cromosoma 3p22.24; la deleción en 6q21 es la segunda anomalía (40-60\%), y también se han identificado trisomía 4, 5, monosomía 8 y supresión del brazo largo del cromosoma $20^{5}$.

Las causas subyacentes de la MW son poco conocidas. El principal factor de riesgo es la gammapatía monoclonal de significado incierto tipo IgM (GMSI-IgM)

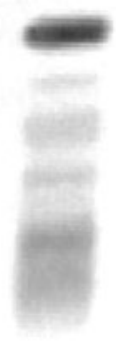

ELP G

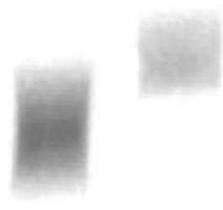

A

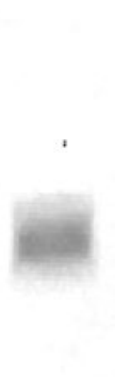

M

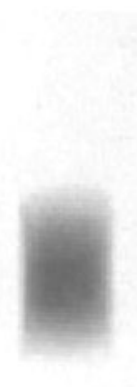

K

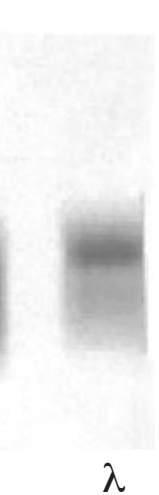

$\lambda$
Figura 3. IFJ con banda en región IgM $\lambda$.

preexistente, pues quienes la padecen tienen hasta 46 veces más probabilidades de desarrollar MW que la población general, con una incidencia acumulada del $10 \%$ a los 5 años, del 18\% a los 10 años y del 24\% a los 15 años. Otros factores de riesgo incluyen antecedentes familiares de MW y otras malignidades de células $\beta^{6}$.

La MW es una enfermedad con curso crónico que puede permanecer estable durante mucho tiempo, por lo que resulta asintomática en la mayoría de los casos. En caso de presentar síntomas, hasta en un 50\% son frecuentes los constitucionales (astenia, anorexia, pérdida de peso), así como la tendencia al sangrado, incluso desde varios años antes del diagnóstico. Los síntomas están relacionados con niveles elevados de IgM y/o infiltración de médula ósea y sitios extramedulares. Las citopenias, que se asocian al reemplazo de las células tumorales, constituyen la presentación clínica más frecuente; la anemia normocítica normocrómica se presenta en el $60-70 \%$ de los enfermos con escasa respuesta reticulocitaria, excepto en los casos en los que también hay hemólisis. En el 20\% de los casos hay trombopenia. Más del $70 \%$ de los enfermos tienen la viscosidad sanguínea elevada, lo cual puede conducir a un deterioro de la microcirculación en el sistema nervioso central, provocando cefalea, visión borrosa, epistaxis y amaurosis en los pacientes con MW. Sin embargo, las manifestaciones clínicas sólo aparecen cuando la viscosidad es de 5 a 10 veces superior a la normal (20-30\% de los casos) ${ }^{1}$. El síndrome de hiperviscosidad es raro si la IgM es mayor de $3 \mathrm{~g} / \mathrm{dl}$, pero no es constante cuando los niveles son superiores. Parece haber una susceptibilidad individualizada para cada paciente, sin que haya una relación exacta entre la tasa de inmunoglobulina con la sintomatología ${ }^{1}$. Las lesiones osteolíticas son muy 
raras $(<1 \%)^{7,8}$, por lo que su presencia obliga a plantearse el diagnóstico diferencial con el mieloma múltiple IgM. La infiltración gastrointestinal también es rara $(<5 \%)$ y requiere un diagnóstico diferencial con otras neoplasias, con frecuencia asociadas a la MW $(7 \%)^{8}$. La actividad de anticuerpos contra proteínas puede dar manifestaciones neurológicas contra glucoproteínas asociadas a mielina, lo que se refleja en polineuropatía, manifestaciones renales con glomerulonefritis por depósito en la membrana basal y endotelio, y amiloidosis $(<5 \%)^{8}$. Algunos pacientes pueden experimentar hepatoesplenomegalia, linfadenopatía y síntomas B.

Otra manifestación de la MW es el síndrome de aglutininas frías, un tipo de anemia hemolítica adquirida debido a la destrucción de los glóbulos rojos por la IgM monoclonal que actúa con mayor eficiencia entre los 4 y $30^{\circ} \mathrm{C}$, con mayor especificidad en contra de los sistemas $\mathrm{I}$ o $\mathrm{HI}$ de los eritrocitos.

El paciente presentado en el caso clínico tuvo datos compatibles con anemia hemolítica, que, si bien se caracteriza por la presencia de anemia normocítica normocrómica, también puede encontrarse macrocítica hipercrómica por el aumento del volumen corpuscular medio indirectamente secundario al incremento en la cantidad de reticulocitos, así como por una falsa elevación del volumen corpuscular medio por las aglutininas frías $^{9}$. En el frotis de sangre periférica son característicos los hematíes en pilas de monedas (fenómeno de Rouleaux), por la paraproteinemia, y pueden observarse linfoplasmocitos.

Para la identificación de la gammapatía monoclonal lgM se cuenta con la electroforesis de proteínas e inmunofijación. En la región $\gamma$ o en la $\beta-\gamma$ aparece el pico monoclonal correspondiente y a una de sus cadenas. El patrón observado en el paciente del caso representa la tendencia de las paraproteínas IgM a formar agregados insolubles y que se precipitan, sobre todo en pacientes con crioglobulinemia, que le da el patrón denominado «M pegajosa» con el pico en la región $\beta-\gamma^{10}$.

Los linfocitos B maduros, linfoplasmocitos y células plasmáticas encontrados son pertenecientes al mismo clon tumoral, lo que le brinda el inmunofenotipo de superficie positiva para IgM, CD19, CD20, CD22, CD25, CD27, FMC7 y negativa para CD103; la expresión de CD5, 10, 23, CD138 puede ser variable. El aspirado suele ser pobre e incluso seco, por aumento de viscosidad y/o hiperplasia reticulocítica, pero debe realizarse en todos los pacientes, incluyendo los ancianos, ya que es un criterio absoluto para su diagnóstico. La célula característica es el linfoplasmocito, que tiene aspecto de linfocito pequeño con contorno ligeramente triangular, citoplasma escaso e hiperbasófilo y núcleo excéntrico con cromatina densa, pero sin distribución en rueda de carro ni nucléolo. A veces tienen inclusiones PAS positivas citoplasmáticas y/o nucleares, denominadas cuerpos de Russell y Dutcher, respectivamente ${ }^{11}$. La naturaleza de estas inclusiones es inmunoglobulínica, que indica la capacidad secretora de estas células. Junto a los linfoplasmocitos puede haber linfocitos maduros y células plasmáticas, que pueden plantear problemas de diagnóstico diferencial con la leucemia linfocítica crónica de células B (LLC-B) y el mieloma múltiple.

Uno de los mayores problemas es la distinción entre MW y GMSI-IgM. Esta última se caracteriza por la presencia asintomática de componente monoclonal IgM con ausencia de infiltración medular, la cual es característica y la única diferencia con la MW asintomática, que como ya se mencionó puede tener un comportamiento semejante al de la GMSI6.

Los casos de MW asintomática con poca probabilidad de progresar son los que tienen una hemoglobina $\geq 12.5 \mathrm{~g} / \mathrm{dl}$ y una $\beta 2-\mathrm{M}$ normal; más de la mitad de ellos no precisará tratamiento a lo largo de seis años de evolución; también hay otras dos entidades que deben tenerse en cuenta en el diagnóstico diferencial: la enfermedad de cadenas pesadas $\mu$ o de enfermedad de Franklin (más próxima a la LLC-B) y el mieloma lgM? En la enfermedad de cadenas $\mu$, la IgM carece de cadenas ligeras, y en el mieloma múltiple IgM hay infiltración medular por células plasmáticas y lesiones osteolíticas, lo que facilita el diagnóstico definitivo.

\section{PRONÓSTICO Y TRATAMIENTO}

El tratamiento se basa en la presencia de síntomas. La GMSI y la MW asintomática no requieren tratamiento ${ }^{12}$, sólo se debe realizar seguimiento cada 3-6 meses, con exploración física (identificar adenopatías y/o hepatoesplenomegalia), inmunoelectroforesis de sangre y orina de $24 \mathrm{~h}$, hemograma y bioquímica con $\beta 2-\mathrm{M}$.

En los casos de MW sintomática, para iniciar el tratamiento se requiere alguno de los siguientes criterios ${ }^{13}$ : 


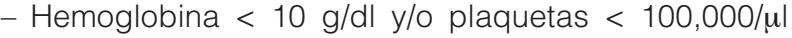
debido a la infiltración medular.

- Síntomas generales o B.

- Crecimiento o síntomas por hepatoesplenomegalia y/o adenopatías.

- Cualquier manifestación asociada a MW: hiperviscosidad, insuficiencia renal, amiloidosis, crioglobulinemia o neuropatía periférica.

Las opciones de tratamiento incluyen rituximab sólo o con alquilantes (bendamustina, ciclofosfamida), inhibidores del proteasoma (bortezomib, carfilzomib), análogos de nucleósidos (fludarabina, cladribina) e ibrutinib (inhibidor de tirosina cinasa de Bruton) ${ }^{12}$.

El rituximab (anticuerpo monoclonal anti-CD20) sólo es adecuado para pacientes con MW y anemia sintomática leve-moderada y para aquéllos en los que la quimioterapia más agresiva es inapropiada, produce tasas de respuesta global (ORR) del 25-40\% con estándar (375 mg/m²/semana durante cuatro semanas) y del 40$60 \%$ con terapia extendida $\left(375 \mathrm{mg} / \mathrm{m}^{2} / \mathrm{semana}\right.$ en las semanas 1-4 y 12-16).

Para pacientes con MW no tratados que experimentan paraproteinemia se recomienda un tratamiento que contenga un inhibidor de proteasoma, como bortezomib, dexametasona y rituximab, o carfilzomib, rituximab y la dexametasona, que resulta ideal.

Para aquéllos que requieren un control inmediato de la paraproteinemia, la plasmaféresis debe realizarse inicialmente. Típicamente, 2-3 sesiones de plasmaféresis reducen los niveles séricos de IgM en un 30-60\%. Los calentadores de sangre deben usarse durante la plasmaféresis en pacientes con crioglobulinemia o aglutinemia fría para prevenir la crioprecipitación y/o la aglutinación de eritrocitos.

Existen terapias de señalización en investigación que incluyen agentes dirigidos a MYD88, CXCR4, BCL2 y CD27/CD70, proteasomas inhibidores y terapia de células $\mathrm{T}$ modificada por receptor de antígeno quimérico (CAR $)^{13}$ :

- Idelalisib bloquea PI3Kס, un factor de transcripción promotor del crecimiento que se activa con MYD88 L265P.
- IMO-8400 es un oligonucleótido que inhibe los receptores Toll 7, 8 y 9, e

- induce apoptosis en las células MYD88 L265P.

- IRAK1/IRAK4 son cinasas que median la señalización hacia NF-kB por parte de MYD88 L265P.

- ABT-199 es un inhibidor BCL-2 (que se encuentra sobreexpresado en la MW) que induce apoptosis.

- Oprozomib e ixazomib son inhibidores de proteasoma con una ORR del 59\%.

- ArgenX-70 es un anticuerpo humano anti-CD70 que bloquea la señalización sCD27-CD70.

- La terapia de células T modificada por un CAR usando un anticuerpo dirigido por CD19 fusionado a cadena $\zeta$ de CD3 y el dominio de señalización intracelular de CD28 (19-28z).

En la segunda línea de tratamiento se puede recurrir a las siguientes opciones ${ }^{13}$ :

- Quimioterapia de alta dosis y trasplante de médula ósea (TMO) autólogo: en estos casos hay que evitar el uso masivo de agentes alquilantes y análogos de purinas antes del TMO.

- Utilizar de nuevo el mismo esquema que en la primera línea si la supervivencia libre de enfermedad desde la finalización del tratamiento es superior a un año.

- Cualquiera de los fármacos de primera línea no usado previamente.

- Otros fármacos: talidomida sola o con dexametasona y pulsos de dexametasona ${ }^{1,13}$.

- La crioglobulinemia responde bien a dosis altas de dexametasona.

Es probable que casi todos los pacientes sufran recaídas o que su enfermedad se vuelva resistente al tratamiento, por lo que los tratamientos de primera línea, dirigidos a paliar los primeros síntomas de la enfermedad, serán desplazados por los de segunda línea a medida que ésta avanza.

Existen una serie de factores pronósticos adversos, como son ${ }^{14,15}$ : 
- Edad $\geq 65$ años.

- Anemia: concentraciones de hemoglobina $<11.5 \mathrm{~g} / \mathrm{dl}$.

- Trombocitopenia: concentraciones de plaquetas $<100,000 / \mathrm{mm}^{3}$.

- $\beta 2$-microglobulina > $43 \mathrm{mg} / \mathrm{l}$.

- Concentración de proteína monoclonal > 470 g/l.

Que implican: bajo riesgo = un factor (excluyendo la edad), con una sobrevida a cinco años del $87 \%$; riesgo intermedio = dos factores o edad mayor de 65 años, con una sobrevida a cinco años del 68\%; o alto riesgo = tres factores o más, con una sobrevida a cinco años del 36\%.

Otros factores pronósticos son:

- Crioglobulinemia.

- Elevada infiltración de la médula ósea por células tumorales.

- Hepatomegalia y linfadenopatías.

- Pérdida de peso.

- Duración de la enfermedad superior a un año.

La supervivencia de los pacientes con más de dos factores de mal pronóstico antes mencionados es mucho menor que la de los pacientes con menos de dos, siendo el caso de nuestro paciente ${ }^{15}$.

En el caso del paciente que presentamos hemos de notar que las manifestaciones iniciales que presentó son demasiado inespecíficas, como lo son generalmente al inicio de esta enfermedad, aunado ello a la anemia hemolítica que presentó a su ingreso, la cual también es una presentación poco frecuente en esta enfermedad. Todo ello dificultó su diagnóstico precoz y tratamiento. La relevancia de este caso se basa en que, a pesar de ser una enfermedad rara y por lo tanto poco diagnosticada, debemos considerarla dentro del protocolo diagnóstico de pacientes con anemia hemolítica que cumplan con otras características epidemiológicas y clínicas de esta enfermedad, y como diagnóstico diferencial, principalmente de mieloma múltiple, ya que a pesar de ser una enfermedad incurable, la calidad y la esperanza de vida pueden mejorar con el tratamiento médico adecuado.

\section{BIBLIOGRAFÍA}

1. García Sanz R, González M, Ocio E. Macroglobulinemia de Waldenström. Servicio de Hematología. Hospital Universitario de Salamanca. Haematologica. 2004;89:89-104.

2. Khosravi Shahi P, Del Castillo Rueda A, Díaz Muñoz de la Espada VM Waldenström's Macroglobulinemia. Servicios de Oncología Médica y Medicina Interna II. Hospital General Universitario Gregorio Marañón. Madrid.

3. Groves FD, Travis LB, Devesa SS, Ries LA, Fraumeni JF Jr. Waldenström's macroglobulinemia: incidence patterns in the United States, 1988-1994.

4. Humphrey JS, Conley CL. Durable complete remission of macroglobulinemia after splenectomy: a report of two cases and review of the literature. Am J Hematol. 1995;48(4):262-71.

5. Takemori N, Hirai K, Onodera R, Kimura S, Katagiri M. Durable remission after splenectomy for Waldenström's macroglobulinemia with massive splenomegaly in leukemic phase. Leuk Lymphoma. 1997;26(3-4):387-43.

6. Dimopoulos MA, Panayiotidis P, Moulopoulos LA, Sfikakis P, Dalakas M. Waldenström's macroglobulinemia: Clinical features, complications, and managment. J Clin Oncol. 2000;18(1):214-26.

7. García-Sanz R, Montoto S, Torrequebrada A, de Coca AG, Petit J, Sureda $A$, et al. Waldenström Macroglobulinaemia: Presenting features and outcome in a series with 217 cases. Br J Haematol. 2001;115(3):575-82.

8. Chen $\mathrm{Cl}$. Treatment for Waldenström macroglobulinemia. Ann Oncol. 2004;15(4):550-8. Review.

9. Vijay A, Gertz MA. Waldenström macroglobulinemia. Blood. 2007;109(12): 5096-103.

10. Alexian R, Weber D. Asymptomatic Waldenström's disease. Semin Oncol. 2003;30:206-10.

13. Ruiz-Argüelles GJ, Ramírez-Cisneros FJ, Flores Martínez J, Cernuda Graham MC. Waldenströjm's macroglobulinemia is infrequent in Mexican mestizos: Experience of a hematological diseases referral center. Rev Invest Clin Mex. 2000;52(5):497-9.

14. Owen RG, Barrans SL, Richards SJ, O'Connor SJ, Child JA, Parapia LA, et al. Waldeström's macroglobulinemia. Development of diagnostic criteria and identification of prognostic factors. Am J Clin Pathol. 2001;116(3):420-8.

15. Kantarjian HM, Alexanian R, Koller CA, Kurzrock R, Keating MJ. Fludarabine therapy in macroglobulinemic lymphoma. Blood. 1990;75(10):1928-32. 